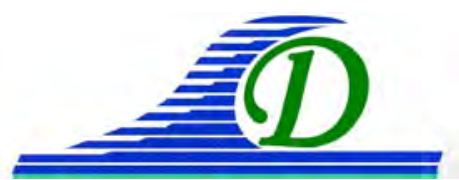

XIII $^{\text {èmes }}$ Journées Nationales Génie Côtier - Génie Civil Dunkerque, 2-4 juillet 2014

DOI: $10.5150 / j n g c g c .2014 .002$ (C) Editions Paralia CFL disponible en ligne - http://www.paralia.fr - available online

\title{
Méthode de décomposition du domaine pour la modélisation numérique d'un jet par la méthode particule-maillage
}

\author{
Khalid ADNAOUI ${ }^{1}$, Nourdine TOUNSI ${ }^{1}$, Mohamed CHAGDALI ${ }^{2}$, \\ Soumia MORDANE ${ }^{2}$
}

1. MITI, Département de Mathématique \& informatique Faculté des Sciences Ben M’Sik, BP 7955, Casablanca. Maroc. khalid.adnaoui@gmail.com

2. LCSM, Département de Physique, Faculté des Sciences Ben M’Sik, BP 7955, Casablanca. Maroc.

\section{Communication non présentée}

\section{Résumé :}

Ce travail porte sur le traitement numérique des problèmes à fortes non linéarités lors de la résolution des équations de Navier-Stokes en particulier celles dues aux fortes recirculations en régime turbulent. L’idée développée consiste à utiliser la méthode de sous domaines. Le domaine dans lequel a lieu l'écoulement est décomposé en plusieurs sous domaines séparés par des frontières fictives. Dans chacun de ces sous domaines, on utilise la méthode numérique la mieux adaptée. Le passage dans tous le domaine se fait par raccordement numérique. Ce raccordement est fait par recouvrement de domaine. Les résultats sont présentés dans le cas d'un jet de rejet émis par le fond dans un canal rectangulaire. Dans cette application on a divisé le domaine d'étude en deux parties : près de la couche limite, on utilise la méthode des différences finies et dans la zone extérieure la résolution est faite par la méthode particulaire. L'interface fictive entre ces deux sous domaines est traitée par la méthode particule-maillage. Une validation de cette approche est faite par une comparaison avec un calcul direct dans tout le domaine. Mots-clés : Equations de Navier-Stokes, Méthodes numériques, Méthodes particulaires, Méthode de différences finies, Décomposition du domaine, Schéma T.S.C de projection interpolation. 
Thème 1 - Hydrodynamique côtière 
XIII ${ }^{\text {èmes }}$ Journées Nationales Génie Côtier - Génie Civil Dunkerque, 2-4 juillet 2014 
Thème 1 - Hydrodynamique côtière 
XIII ${ }^{\text {èmes }}$ Journées Nationales Génie Côtier - Génie Civil Dunkerque, 2-4 juillet 2014 
Thème 1 - Hydrodynamique côtière 
XIII ${ }^{\text {èmes }}$ Journées Nationales Génie Côtier - Génie Civil Dunkerque, 2-4 juillet 2014 
Thème 1 - Hydrodynamique côtière 